Conclusion All patients received a first line antibiotic in line with Trust guidance, although no patients had confirmed intracranial infection. Pus samples did not alter antibiotic choices in this small cohort. This suggests, in our population, most patients can be safely managed with co-amoxiclav as first line therapy.

\section{G146(P) EFFORTS TO INCREASE EARLY DETECTION OF OBESE AND OVERWEIGHT CHILDREN IN GENERAL PEDIATRIC CLINICS}

S Khan, S Osman, Z Kanfosh, M Al Malaheem, M Laspinas, M Suello, E Joseph, J Martinez. Pediatrics, Hamad Medical Corporation, Doha, Qatar

\subsection{6/archdischild-2020-rcpch.117}

Aim Lack of attention to the pediatric Body Mass Index (BMI) by the physician during outpatient visits decreases the early detection of obese and overweight children visiting the clinic for reasons other than increased weight and subsequently delays starting active management. The aim of this project was to promote early detection and management of patients with a BMI above or equal to 85 th percentile for age and gender (High BMI).

Methods Several Plan Do Study Act cycles were implemented:

- Education all physician and the nursing staff regarding measurement of BMI in children

- To choose the 'office visit' template on Cerner (electronic medical record) so that the BMI is automatically built into the physician note

- Nursing Staff identified Patients with high BMI during vital signs check and placed a reminder next to the patient's room number to alert the treating physician about patient's high BMI status

- High BMI Patients were provided with an educational pamphlet on diet management and referred to dietician

Results

Conclusion The recognition of BMI is the first step to early detection of pediatric obesity. Through simple sustained efforts we were able to improve the management of High BMI children by improving counseling and dietician referral to above $75 \%$ in the last quarter of 2018 . We intend to follow the patients detected to be overweight and obese in these clinics to see if simple measures like family counselling and dietician referral have resulted in BMI reduction over time.

\section{G147(P) SUSTAINED ENHANCEMENT OF TEAM LEARNING TWO YEARS AFTER IMPLEMENTING A SIMPLE INITIATIVE}

${ }^{1} \mathrm{H}$ Coleman, ${ }^{1} \mathrm{~S}$ Milne, ${ }^{1} \mathrm{~V}$ Rae, ${ }^{1} \mathrm{~K}$ Jarman, ${ }^{1} \mathrm{~F}$ McQuaige, ${ }^{2} \mathrm{~N}$ Bee, ${ }^{1} \mathrm{~S}$ Joseph, ${ }^{1} \mathrm{M}$ Stark. ${ }^{1}$ Acute Receiving Unit, Royal Hospital for Sick Children, Edinburgh, UK; ${ }^{2}$ Emergency Department, Royal Hospital for Children, Glasgow, UK

\subsection{6/archdischild-2020-rcpch.118}

Background We identified significant challenges to longitudinal team learning in the Acute Receiving Unit of our quaternary paediatric hospital. These included shift work, trainee rotation and a high service demand. We hypothesised that a regular 'Learning Points' email would be a simple and cost-free way to address this. Since establishing this departmental project in 2017 we have sought to improve and expand throughout the hospital.

Aims Our key aims were to:

- Inspire and enhance team work and learning - both within and between departments

- Promote evidence-based practice

- Promote a culture of quality improvement and patient safety, highlighting local and national initiatives.

Methods Regular emails were compiled by a small group of paediatric trainees. Content included interesting cases, clinical teaching, quality improvement and patient safety information. An initial electronic survey in January 2018 yielded positive feedback and identified areas to improve. Subsequently circulation was widened to include staff from other departments (consultants, senior nursing staff, paediatric ST1-8, GP and FY trainees). Contributions were invited from recipients. The email template was updated and changed to PDF form to enable e-portfolio use. A follow-up electronic survey was disseminated in July 2019 to re-evaluate our intervention. This included space for free-text comments. Questions included:

- Do you find the learning points email a useful learning resource? If so, can you explain what the key benefits of it are?

\begin{tabular}{|c|c|c|c|c|c|c|c|c|}
\hline \multirow[t]{3}{*}{ Pre-intervention } & 2017 & 2017 & 2017 & 2017 & 2018 & 2018 & 2018 & 2018 \\
\hline & 1st & 2nd & $3 r d$ & 4th & $1 s t$ & 2nd & $3 r d$ & 4th \\
\hline & Quarter & Quarter & Quarter & Quarter & Quarter & Quarter & Quarter & Quarter \\
\hline $47.3 \%$ & $81 \%$ & $88.1 \%$ & $87.9 \%$ & $77 \%$ & $85 \%$ & $87.5 \%$ & $78.7 \%$ & $91.6 \%$ \\
\hline
\end{tabular}

Abstract 146(P) Table 1 Percentage of patients with High BMI who received counseling and dietician referral

\begin{tabular}{|c|c|c|c|c|c|c|c|c|}
\hline \multirow[t]{3}{*}{ Pre-intervention } & 2017 & 2017 & 2017 & 2017 & 2018 & 2018 & 2018 & 2018 \\
\hline & 1st & 2nd & $3 r d$ & 4th & 1st & 2nd & $3 r d$ & 4th \\
\hline & Quarter & Quarter & Quarter & Quarter & Quarter & Quarter & Quarter & Quarter \\
\hline $33.3 \%$ & $54.7 \%$ & $43.3 \%$ & $44.6 \%$ & $65.3 \%$ & $63.3 \%$ & $45.3 \%$ & $48.3 \%$ & $76.6 \%$ \\
\hline
\end{tabular}

